\title{
Comparative Study of Ear Prominence with and without Placing Conchomastoid Suture Using Post Auricular Muscle as an Anatomical Landmark - A Randomised Clinical Trial
}

\author{
Anagha Yogesh Rajguru1, Sanket Chandrashekhar Prabhune², Khaleel Mannuru Basha ${ }^{3}$, \\ Lakshmi Sravya Yarlagadda ${ }^{4}$, Kaenat Ahmed ${ }^{5}$ \\ 1, 2, 3, 4, 5 Department of Otorhinolaryngology, Krishna Institute of Medical Sciences \\ (Deemed to Be University), Karad, Maharashtra, India.
}

\section{ABSTRACT}

\section{BACKGROUND}

Majority of the people undergoing tympanoplasty done by using postauricular incision face the cosmetic problem of ear prominence. The auricularis posterior muscle acts as a vector while placing sutures between the conchal cartilage and mastoid fascia and helps to reduce ear prominence post tympanoplasty. We wanted to study the difference in prominence of ear with and without conchomastoid suture placement post tympanoplasty done using post auricular incision. Evaluate the importance of the posterior auricular muscle as an anatomical landmark by placing conchomastoid suture during anatomical reduction of ear prominence post tympanoplasty and evaluate the usefulness of conchomastoid suture during anatomical reduction of ear prominence post tympanoplasty.

\section{METHODS}

A total of 60 clinically diagnosed cases of chronic suppurative otitis media tubotympanic type requiring tympanoplasty were included in our study. All cases were divided in two groups viz. i and ii randomly by first come first method and were subjected to tympanoplasty with and without conchomastoid suture placement during closure respectively. During follow up period, the prominence of ear pinna was assessed till $10^{\text {th }}$ postoperative day in all cases.

\section{RESULTS}

In this study, ear prominence was present in $10 \%$ (3) of the cases with conchomastoid suture placement after tympanoplasty i.e. group i and in $33 \%$ (11 cases) without conchomastoid suture i.e. group ii after tympanoplasty. $\mathrm{P}$ value is 0.01461 which is significant.

\section{CONCLUSIONS}

From this study it can be concluded that with conchomastoid suture placement we have better results of healing with less ear prominence post tympanoplasty rather than without conchomastoid sutures.

\section{KEY WORDS}

Tympanoplasty, Chronic Suppurative Otitis Media, Conchomastoid Suture
Corresponding Author:

Dr. Sanket Chandrashekhar Prabhune, Department of Otorhinolaryngology, Krishna Institute of Medical Sciences (Deemed to Be University), Karad, Maharashtra, India.

E-mail: sanketprabhune5@gmail.com

DOI: $10.14260 / \mathrm{jemds} / 2020 / 866$

How to Cite This Article:

Rajguru A.Y, Prabhune S.C, Basha K.M, et al. Comparative study of ear prominence with and without placing conchomastoid suture using post auricular muscle as an anatomical landmark - a randomized clinical trial. J Evolution Med Dent Sci 2020;9(52):3963-3967, DOI: $10.14260 / \mathrm{jemds} / 2020 / 866$

Submission 12-09-2020,

Peer Review 04-11-2020,

Acceptance 11-11-2020,

Published 28-12-2020.

Copyright (c) 2020 Anagha Yogesh Rajguru et al. This is an open access article distributed under Creative Commons Attribution License [Attribution 4.0 International (CC BY 4.0)] 


\section{BACKGROUND}

It is important to know the detailed anatomy of external ear before surgical correction of the prominent ear pinna. The external ear consists of fibro elastic cartilage which is covered by perichondrium. Anteriorly, skin is adherent to the perichondrium. As loose areolar tissue is present posteriorly, the skin is less adherent posteriorly to perichondrium as compared to anterior. The lobule is a flappy structure of ear pinna as it is devoid of cartilage but it contains thick skin and loose connective tissue. ${ }^{1}$

The anatomic elements of the ear are the root of the helix, helix, antihelix, superior (posterior) crus of antihelix, inferior (anterior) crus of antihelix, tragus, antitragus, triangular fossa, scaphoid fossa, concha cymba, concha cavum, and lobule. The extrinsic muscles of the auricle are the anterior, superior, and posterior auricular muscles. One of the most common aesthetic problem post tympanoplasty done by using post auricular incision is ear prominence. The ear surgeries are done since seventh century and can be found in the Ayurvedic literatures which were documented by Indian author Sushruta. ${ }^{2}$ The reduction of protruding ears by the correction of deformities of pinna has been the objective of many techniques described by Luckett et al. $^{3}$ in 1910 . There are majorly three ear correction techniques which are broadly divided into cartilage-sculpting and cartilage-sparing and combination of both. More specifically, they are cutting, scoring, and suturing techniques, incision less otoplasty and implants, of which suturing techniques are easy with less recurrence rate. ${ }^{4}$

Suture-based techniques used more commonly in reduction of ear prominence in comparison with cartilage scoring as they minimize damage to the perichondrium and the possibility of cartilage necrosis. ${ }^{5}$ Reduction of ear prominence by suturing was first described by Owens and Delgado et al., which was modified later by Furnas et al.,5 by placing non-absorbable mattress sutures between the conchal cartilage and mastoid fascia. The basic principle of most suturing techniques used in reduction of ear prominence is to make a post auricular incision and the insertion of absorbable or non-absorbable sutures for addressing the relative obtuse antihelical rim and upper crus, as well as the anterior rotation of the conchal bowl. 5 For avoidance of error while reduction of ear prominence two separate rows of sutures are placed in individual correction of ear prominence. While reduction of ear prominence by suturing technique is sometimes challenging, especially placing sutures over mastoid fascia as it is not a precise anatomical landmark. A correct anatomical vector is a need while doing reduction of ear prominence by suture technique or else it leads to malrotation of ear. The auricularis posterior muscle or post auricular muscle is visualized in dermo-fascial flap ear reduction technique posteriorly towards mastoid as dissection proceeds. The posterior auricular muscle consists of two or three fleshy fasciculi, which arise from the mastoid portion of the temporal bone by short aponeurotic fibers. They are inserted into the lower part of the cranial surface of the concha ${ }^{6}$ Its physiological function is to adduct the pinna. Ear prominence reduction is based on two points or areas of which conchal bowl is the one which is most of the times hypertrophied and or the other one which is antihelix which may be flattened or underdeveloped. The cosmetically better pinna projects approximately $20^{0}$ to $30^{\circ}$ from the skull. The fully developed ear measures approximately 50 to $60 \mathrm{~mm}$ in length and nearly 50 percent of its length in width and along its vertical axis, the ear is a gentle $20^{0}$ to $25^{0}$ posteriorly. ${ }^{7}$ To measure the distance from helix of the ear pinna to scalp is a better method of assessing the ear prominence which is measured by 3 points using distance between superior point of helix of ear pinna, the midpoint of helix and lobule of ipsilateral ear which are approximately 10 to $12 \mathrm{~mm}, 16$ to 20 $\mathrm{mm}$, and 20 to $22 \mathrm{~mm}$ respectively. These measurements serve as important key points in bilateral reduction of ear pinna for aesthetically better ear pinna and for purpose of bilateral symmetry. While in the unilateral reduction of ear pinna, measurements from the aesthetically better ear can be used as key point for reduction of contralateral ear pinna prominence. ${ }^{8}$ Post auricular muscle plays an important role in contour of ear pinna and any anatomical variation in it produces its prominence according to Smith and Takashima et al. ${ }^{9}$ The posterior auricular muscle is one of the three extrinsic muscles of the external ear. It has been discussed earlier that this muscle helps in positioning of ear pinna in accordance with cranial surface. It has been studied that post auricular muscle is a major factor in deciding ear prominence and electrical activity in this muscle is in relation to certain facial movements. ${ }^{9}$ Results of another study demonstrate a linear relationship between the posterior auricular muscle insertion site and ear projection.10 The unilateral reduction of ear prominence is often more challenging in achieving aesthetic results and postoperative symmetry, compared to bilateral procedures. The increased difficulties consist of anatomical deformities of the unilaterally prominent ear and predefined necessity to replicate the no prominent ear. ${ }^{11}$

In unilateral reduction of ear prominence for both cosmetic outcomes and early complication rates, post auricular suturing techniques seem to be a superior option and it is likely to be particularly beneficial for younger patients in whom short-term complications are less well tolerated. ${ }^{12}$ In this study, we will observe the difference in prominence of ear with and without conchomastoid suture placement after tympanoplasty done by making post auricular incision by comparing with the contralateral non-operated aesthetically better ear. It will be a unique study done first time in rural tertiary set up of western Maharashtra.

\section{METHODS}

This is a randomised clinical trial was carried out in a tertiary care hospital in rural Western Maharashtra. The ethical clearance from institutional ethics committee was sought vide their letter number KIMSDU/IEC/09/2019 dated $14 / 12 / 2019$. The institutional ethics committee of Krishna Institute of Medical Sciences, Karad and KIMSDU, Karad (IEC Karad) is registered with DCGI vide letter number ECR/708/Krishna/Inst/MH/2013 dated 08.05.2013. Prior informed consent was obtained from all the participants in this study. As this study was of short duration, it was planned with at least 25 cases in each group. However, keeping the possibilities of drop out during follow up in the study, it was decided to enrol minimum 30 cases in each group, and hence, the sample size was 60 ( 37 females, 23 males). 
All clinically diagnosed cases of tubotympanic type chronic suppurative otitis media of all age group (more than 15 years) irrespective of their gender requiring tympanoplasty were included in the study. Patients having active ear discharge, bleeding disorders, less than 15 yrs. of age, active menstruation, haemoglobin level less than $10 \mathrm{gm} / \mathrm{dl}$ were excluded. All cases were randomised by lottery method into group I, II and subjected to tympanoplasty with and without conchomastoid suture placement respectively. Routine blood and urine examination viz. complete blood count, bleeding time, clotting time, blood- grouping as well as urine routine and microscope etc. was undertaken in every case before operation. During closure of incision post tympanoplasty, after identifying auricularis posterior muscle as an anatomical landmark conchomastoid mattress sutures were placed with Vicryl 4 - 0 in group I cases. Clipping the first stitch to allow passage of subsequent stitches was done. Careful tensioning of the knots was done to provide controlled and anatomical reduction of ear prominence with a natural aesthetic outcome. Post auricular muscle was used as an anatomical landmark here while placing the sutures. In group II cases no conchomastoid sutures were placed while suturing post auricular incision after tympanoplasty. Meticulous technique, appropriate preoperative planning and close post-operative care were used to avoid any complication. During follow up period of $10^{\text {th }}$ day, the prominence of ear pinna on operated ear was compared with non-operated ear in all cases.

Recurrence of ear prominence was noted in group I cases and those cases were posted for other techniques like cartilage-cutting techniques or anterior scoring or burring etc. of otoplasty by obtaining prior consent. While cases having ear prominence in group II were posted for ear prominence reduction technique (otoplasty) by placing conchomastoid suture using Vicryl 4 - 0 suture material obtaining prior consent and followed up after 10 days for assessment of recurrence rate.

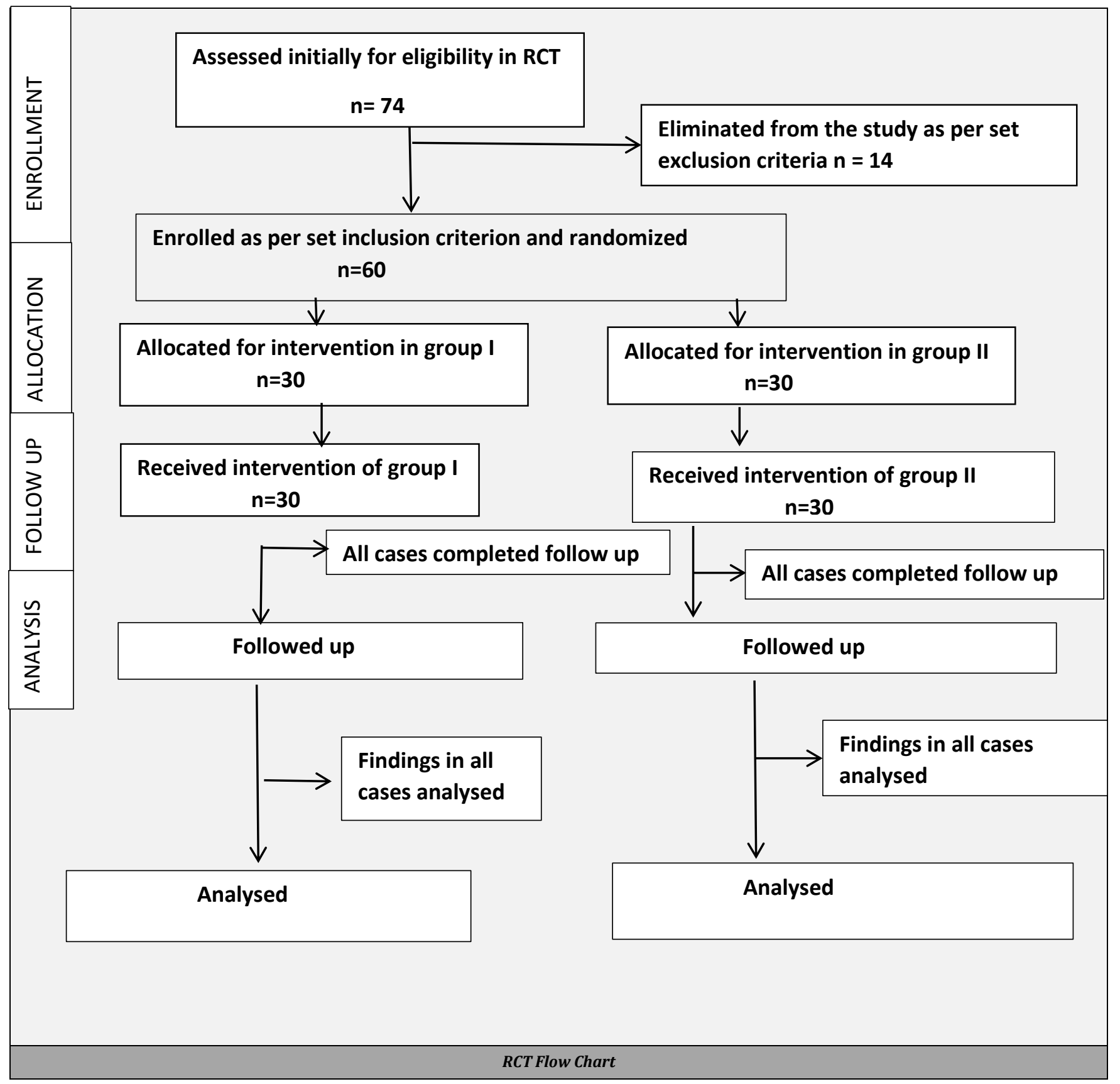




\section{Statistical Analysis}

Chi square test was the statistical method used here for comparison of prominence of ear pinna between the two groups and the software used was In Stat 9.0, India.

\section{RESULTS}

Initially 74 cases were willing for enrolment in the study. In those 14 cases were excluded according to set exclusion criteria. Finally, this study was done over 60 clinically diagnosed cases in age range of 15 to 60 years, M: F ratio was 23:37, mean age of group-I and II was $33.5 \pm 12.87$ and 37.42 \pm 12.62 respectively and $\mathrm{M}: \mathrm{F}$ ratio in group I was $11: 19$ and group II was 2:3 (Table 1).

\begin{tabular}{|cccc|}
\hline Parameters & All Groups $\mathbf{n}=\mathbf{6 0}$ & Group I n = 30 & Group II n = 30 \\
Mean \pm SD & $35.31 \pm 12.77$ & $33.5 \pm 12.87$ & $37.42 \pm 12.62$ \\
Males & \multicolumn{3}{c}{ Sex Ratio } \\
Females & 23 & 11 & 12 \\
\hline \multicolumn{4}{c}{ Table 1. Demographic Data } \\
\hline
\end{tabular}

In our study the ear pinna prominence is present in $10 \%$ (3) of the cases with conchomastoid suture placement i.e. in group I and in $33 \%$ (11 cases) without conchomastoid suture placement i.e. group II after tympanoplasty. Therefore $90 \%$ (27 cases) with conchomastoid suture placement i.e. group I, didn't need further correction or revision surgery for reducing ear prominence, whereas $67 \%$ (19) of the cases didn't require correction in group II. After applying chi square test, we obtained $p$ value 0.01461 which is significant (Table 2).

No post-operative early or late complications like haematoma, hypertrophic scars, keloids, infection, perichondritis, suture complications, cartilage or skin necrosis were noted in any of the case.

In group I, 3 cases were having ear prominence for which anterior scoring technique was done in all of them while 11 cases in group II having ear prominence were posted for suture otoplasty where conchomastoid suture placement was done. Ear prominence recurrence occurred in 1 case in group II post conchomastoid suture placement,

Recurrence rate after conchomastoid suture placement was $10 \%$ as 3 out of 10 and 1 out of 11 cases had ear prominence.

\begin{tabular}{|ccccc|}
\hline $\begin{array}{c}\text { Ear } \\
\text { Prominence }\end{array}$ & Group I & Group II & Chi Square & P Value \\
Present & $3(10 \%)$ & $11(33 \%)$ & 5.9627 & $0.01461^{*}$ \\
Absent & $27(90 \%)$ & $19(67 \%)$ & \\
\hline \multicolumn{5}{c}{ Table 2. Chi Square Test } \\
\hline${ }^{*} \mathrm{p}<0.05$ is significant
\end{tabular}

\section{DISCUSSION}

Suture-based techniques are most commonly used in reduction of ear pinna prominence as they minimize damage to the perichondrium which can cause cartilage necrosis. Traditionally, surgeons have used conchoscaphal sutures to correct effacement of the antihelical fold or conchomastoid sutures with or without cartilage excision. We have unified these techniques allowing a simple suture-based technique that can minimize commonly encountered complications like cartilage necrosis and over or under correction of ear pinna prominence.

The advantage of taking small sequential bites with the conchomastoid sutures is that bowstringing of the thread. Once the placement of conchomastoid sutures is done the surgeon can assess the correction needed comparing with other non-operated ear and then by tying the threads down. This allows for proper aesthetic reduction of ear prominence avoiding over or under correction. As knots are visualised when placed superficially, they are placed deep near mastoid. Kenneth J. Stewarth et al. states that when sutures, especially mattress sutures if widely placed can result in transverse pull which causes deformity in cartilage and can lead to malrotation of ear pinna. ${ }^{3}$

Smaller bites and increased number of suture knots can be preferred as it can provide aesthetically better reduction of ear pinna. Another method of placing second row of sutures between concha and premastoid fascia can be done which can also provide aesthetically better ear according to desired rotation. Accurate placement of sutures on the conchal lateral wall is a must. Excision of cartilage is required when the concha is stiff or prominent as anterior rotation of pinna is alone insufficient in such cases which is a rare entity. ${ }^{3}$

Nicolétis and Guerin-Surville et al. first stated the use of posterior auricular muscle in reduction of ear prominence by transposition of muscle in 1978.10 Guyuron and Deluca et al. described the importance of the post auricular muscle and its insertion in deciding the contour or prominence of ear pinna. ${ }^{11}$ More recently, techniques involving transposition of the insertion of the muscle have been described in accordance with Azad S and Scuderi N et al.12,13

However, the employment of this muscle as a landmark in post tympanoplasty sutures has not been documented previously according to C Scuderi N et al. and Stephen et al.14,15 No any post-operative early or late complication was noted in this study which is in accordance with study done by Ethan B. Handler et al. which states that if sincere efforts, proper technique and accurate preoperative planning is executed the complications in technique of ear prominence reduction is rare. ${ }^{16}$

In this study, recurrence rate was $10 \%$ both in group I and II which was in accordance with study done by kenneth J. Stewarth et al. which states that cases undergoing posterior suturing technique for ear pinna prominence reduction uncorrected hypertrophic conchal bowl is a major cause of recurrence which accounts for $10 \%$ of recurrence. ${ }^{3}$

Another study by Ethan B. Handler ${ }^{17}$ states that the nonabsorbable suture material like Prolene and nylon have a great risk of erosion of skin and underneath tissue causing deformity in post auricular region and also causing malrotation of pinna by tendency of slipping for which secondary otoplasty is required, while use of absorbable suture material like Vicryl and catgut more commonly causes infection and granuloma formation as they are more reactive. ${ }^{16}$ In cases having inflammatory reaction or extrusion due to non-absorbable suture material, removal of the suture resolves the complication, though the final result may be compromised. ${ }^{1}$ So, in this study we have used synthetic absorbable suture material like Vicryl 4 - 0 to avoid such complications. 
The usefulness of conchomastoid suture placement using posterior auricularis muscle as a landmark is done in the present study. It will be more helpful for proper closure of tympanoplasty incision without ear pinna prominence and aesthetically better ear.

\section{CONCLUSIONS}

Conchomastoid suture placement gives better results of healing with less ear prominence i.e. aesthetically better ear post tympanoplasty done by post auricular incision. It also has fewer complications and lower recurrence rate as compared to non-placement of conchomastoid sutures. It will be beneficial for patients undergoing tympanoplasty done by using post auricular incision as it will provide an aesthetically better ear.

Data sharing statement provided by the authors is available with the full text of this article at jemds.com.

Financial or other competing interests: None.

Disclosure forms provided by the authors are available with the full text of this article at jemds.com.

\section{REFERENCES}

[1] Banks C, Cheney M. Open access atlas of otolaryngology, head \& neck operative surgery. http://campus.cerimes.fr/orl/cycle3/otoplasties.pdf

[2] Hauben DJ. Sushruta Samhita (Sushruta'a Collection) (800-600 B.C.?). Pioneers of plastic surgery. Acta Chir Plast 1984;26(2):65-8.

[3] Rogers B0. The classic reprint. A new operation for prominent ears based on the anatomy of the deformity by Luckett WH. (reprinted from Surg Gynec \& Obst 10: 6357, 1910). Plast Reconstr Surg 1969;43(1):83-6.
[4] Stewart KJ, Lancerotto L. Surgical otoplasty: an evidencebased approach to prominent ears correction. Facial Plast Surg Clin North Am 2018;26(1):9-18.

[5] Nikkhah D, Farid A, Sadri A, et al. A scaphomastoid sutures technique for prominent ear otoplasty. Plast Reconstr Surg Glob Open 2018;6(9):e1892.

[6] Janis JE, Rohrich RJ, Gutowski KA. Otoplasty. Plast Reconstr Surg 2005;115(4):60e-72e.

[7] Bendrihem R, Vacher C, Barbet JP. The posterior muscles of the auricle: anatomy and surgical applications. Ann Otolaryngol Rhinol 2015;2(1):1020.

[8] Papel ID. Facial plastic and reconstructive surgery. $3^{\text {rd }}$ edn. New York: Thieme 2009.

[9] Janz BA, Cole P, Hollier LH, et al. Treatment of prominent and constricted ear anomalies. Plast Reconstr Surg 2009;124(Suppl 1):27e-37e.

[10] Smith DW. Protruding auricle: a neuromuscular sign. Lancet 1978;(8067):747-9.

[11] Nicolétis C, Guerin-Surville H. Prominent ears: transposition of the posterior auricular muscle on the scapha: a new technique. Aesthetic Plast Surg 1978;2(1):295-302.

[12] Guyuron B, DeLuca L. Ear projection and the posterior auricular muscle insertion. Plast Reconstr Surg 1997;100(2):457-60.

[13] Szychta P, Stewart KJ. Comparison of cartilage scoring and cartilage sparing techniques in unilateral otoplasty a tenyear experience. Ann Plast Surg 2013;71(5):522-7.

[14] Azad S, Edwin A, Kumar PV. Posterior auricular muscle--a useful adjunct in otoplasty. $\mathrm{Br} \mathrm{J}$ Plast Surg 2003;56(7):722-3.

[15] Scuderi N, Tenna S, Bitonti A, et al. Repositioning of posterior auricular muscle combined with conventional otoplasty: a personal technique. J Plast Reconstr Aesthet Surg 2007;60(2):201-4.

[16] Stephen C, Lowrie AG. The posterior auricular muscle: a useful anatomical landmark for otoplasty. J Laryngol Otol 2016;131(5):465-7.

[17] Handler EB, Song T, Shih C. Complications of otoplasty. Facial Plast Surg Clin North Am 2013;21(4):653-62. 